\title{
RDUS
}

Revue de DROIT UNIVERSITÉ DE SHERBROOKE

Titre : $\quad$ LES INCERTITUDES JURIDIQUES DE L'IDENTITÉ RELIGIEUSE

Auteur(s): $\quad$ Vincente FORTIER

Revue: $\quad$ RDUS, 2007-2008, volume 38, numéro 2

Pages: $\quad 385-411$

ISSN : $\quad 0317-9656$

Éditeur : $\quad$ Université de Sherbrooke. Faculté de droit.

URI : $\quad$ http://hdl.handle.net/11143/11585

DOI : $\quad$ https://doi.org/10.17118/11143/11585 
Page vide laissée intentionnellement. 


\title{
ARTICLE
}

\section{LES INCERTITUDES JURIDIQUES DE L'IDENTITÉ RELIGIEUSE}

\author{
par Vincente FORTIER *
}

Si le nom, le prénom, la date de naissance, la filiation mais aussi les caractéristiques physiques sont des éléments de l'identité d'un individu et permettent de le singulariser, la religion contribue également à la construction de l'identité personnelle.

Or l'identité civile telle qu'elle est appréhendée par le droit et l'identité humaine, structurée autour d'une foi plus ou moins intense, semblent mener une existence parallèle puisque le droit français en principe est indifférent aux préférences religieuses des individus.

Cependant l'être humain n'est pas réductible à ses seuls attributs légaux. Comment, dès lors, concilier les points de vue juridique et ontologique afin de prendre en compte le religieux dans l'humain?

Dans certains cas, l'identité religieuse cherche un prolongement dans l'identité juridique, civile ou physique. Dans d'autres hypothèses, plus problématiques, le croyant demande un affichage du religieux dans l'espace public.

If the surname, given names, date of birth, filiation as well as physical characteristics are elements for determining a person's specific identity, then logically, religion must also be a factor contributing to the establishment of one's identity.

However, civil identity, as determined by law, and human identity generally, which may be more or less influenced by one's religious faith, seem to exist in parallel in France since French law, as a rule, is indifferent to the religious preferences of individuals.

Nevertheless, a human being cannot be simply reduced to the sum of his or her legal attributes. How therefore would it be possible to reconcile both the legal and ontological aspects of human existence in order to take into account a person's religion?

In certain situations, religious identity merits inclusion as an element as pertinent as a person's legal, civil or physical identity. Religious identity becomes more problematic in a lay society when its adherents demand that there be displays of religious symbols in public areas or premises.

* . Directrice de recherche au CNRS, Université de Montpellier I, UMR 5815, "Dynamiques du droit". 


\section{SOMMAIRE}

I - Affirmer son identité religieuse pour affermir

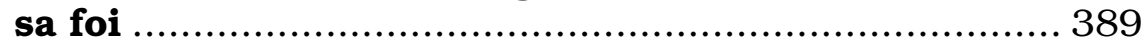

A - L'identité civile saisie par la religion.................... 390

B - L'identité corporelle saisie par la religion ............ 397

II - Revendiquer son identité religieuse pour afficher sa foi ...................................................... 402 
(2008) 38 R.D.U.S.

Les incertitudes juridiques

de

387

l'identité religieuse

L'identité ne se compartimente pas, écrit Amin Maalouf dans Les identités meurtrières, elle ne se répartit ni par moitiés, ni par tiers, ni par plages cloisonnées. Je n'ai pas plusieurs identités, j'en ai une seule, faite de tous les éléments qui l'ont façonnée, selon un "dosage" particulier qui n'est jamais le même d'une personne à l'autre. ${ }^{1}$

L'identité, c'est donc ce qui fait qu'une personne est ellemême et non une autre; ce qui permet de la reconnaître et de la distinguer des autres ${ }^{2}$, l'ensemble des caractères qui concourent à sa singularité. Identité civile et identité physique forment cet ensemble de caractéristiques qui font qu'un être humain existe comme être unique et ne se confond avec aucun autre.

Le nom, le prénom, la date de naissance, la filiation, mais aussi le corps, l'enveloppe charnelle, sont les éléments de l'identité que le droit connaît. Mais au-delà des éléments de son état au sens du droit, ce qui contribue à façonner l'identité d'un individu, c'est-à-dire ce sentiment d'être par lequel il éprouve que son moi le distingue radicalement des autres" ${ }^{3}$, ce sont "toutes les caractéristiques morales, intellectuelles, spirituelles, physiques ou même socioprofessionnelles qui le distinguent des autres". La religion contribue incontestablement à la construction de l'identité personnelle, que celle-ci soit reçue en héritage ou qu'elle procède d'une quête spirituelle autonome. Identité humaine, structurée autour d'une foi plus ou moins impérialiste, et identité civile qui appréhende le sujet de droit de façon utilitaire, semblent mener une existence parallèle, puisque le droit français est, en principe, indifférent aux préférences religieuses des individus, à leur vie intérieure, dans le respect bien compris des principes d'égalité et de neutralité. Et du reste, lorsque le droit prend en compte la

1. Amin Maalouf, Les identités meurtrières, Paris, Le Livre de poche, 2007 à la p. 8.

2. Gérard Cornu, Vocabulaire juridique, 8e éd., Paris, PUF, 2007, s.v. «identité».

3. Alain Bernard, "L'identité des personnes physiques en droit privé, remarques en guise d'introduction" dans Jacques Chevalier, dir., L'identité politique, Paris, PUF, 1994, 127. 
religion, c'est pour affermir le principe d'égalité, nommant alors pour protéger selon les mots de Danièle Lochak ${ }^{4}$.

Dès lors, si nous considérons que l'être humain n'est pas réductible à ses seuls attributs légaux, mais que, par ailleurs, le droit ignore le divin, comment concilier les points de vue juridique et ontologique afin de prendre en compte le religieux dans l'humain?

C'est sur deux modes, nous semble-t-il, que le religieux peut chercher à s'exprimer, comme élément de l'identité. Un mode mineur tout d'abord, intime, à dimension plus personnelle que collective lorsqu'il s'agit seulement pour le croyant d'affirmer son identité religieuse en investissant les différentes composantes de son identité juridique ${ }^{5}$. Un mode majeur, ensuite, lorsque la religion apparaît comme un corpus de représentations, de croyances et de pratiques dont la prétention normative vient concurrencer voire supplanter le système juridique. Il ne s'agit plus ici d'affirmer sa religiosité comme contribuant à l'identité, mais de la revendiquer comme élément premier de son identité.

L'une et l'autre hypothèses entrent dans le champ du droit, moins fortuitement que l'on pourrait le croire, le plus souvent par sollicitation du citoyen-croyant. Dans le premier cas, l'identité religieuse cherche un prolongement, une prise en compte dans l'identité juridique, civile ou physique. Dans le second cas, la religion et son vécu sont en eux-mêmes l'expression d'une recherche identitaire: le croyant semble s'inscrire dans une logique d'opposition ou d'incompatibilité avec le droit (ou bien citoyen, ou bien croyant) en ce qu'il demande un affichage du religieux dans l'espace public.

\footnotetext{
4. Danièle Lochak, "L'autre saisi par le droit" dans Bertrand Badie et Marc Sadoun, dir., L'autre, Paris, Presses de la fondation nationale des sciences politiques, 1996, 179.

5. Pour de plus amples développements sur ce thème nous renvoyons à la thèse de doctorat de Myriam Pendu, Le fait religieux en droit privé, Rennes, 2004 aux pp. 31 et s.
} 
(2008) 38 R.D.U.S.

Les incertitudes juridiques

de

389

l'identité religieuse

\section{I - Affirmer son identité religieuse pour affermir sa foi}

Appréhendée largement, l'identité d'une personne est constituée de multiples critères qui renvoient à ce qu'elle est, à son caractère, sa personnalité, son image, sa réputation ou encore à ses origines, culturelles, géographiques, sociales ${ }^{6}$. L'identité est en ce sens multidimensionnelle et polymorphe ${ }^{7}$. Entendue plus strictement et du point de vue du droit civil, l'identité d'une personne est révélée par certains éléments qui constituent son état (individuel et familial) et qui sont destinés à singulariser l'individu en tant que sujet de droit et à déterminer sa situation personnelle au regard de la loi civile. Prolongeant cette individualité de l'être humain, le corps, en tant que support matériel de la personnalité, "substratum de la personnalité juridique" selon les termes de Carbonnier, est également un facteur d'identité.

Les référents identitaires mis en place par le droit sont dénués de toute dimension religieuse. S'ils sont au premier chef des instruments de police civile, ils revêtent cependant une dimension personnelle et affective, rattachant la personne à un groupe social et évoquant son histoire familiale comme dans le cas du nom ${ }^{8}$. Dès lors, ces référents identitaires ne doivent pas seulement être appréhendés dans leur fonction sociale : investis par ceux qu'ils désignent, ces référents vont se charger d'un sens qui dépasse leur stricte dimension technique et vont jouer le rôle de révélateurs d'une réalité plus subjective de leur être ${ }^{9}$. Le droit dans une certaine mesure permet cette réappropriation par l'individu de son identité et la mise en adéquation de son identité juridique avec ses aspirations profondes et pour ce qui nous occupe, avec ses convictions religieuses.

6. David Bakouche, Droit civil, les personnes, la famille, Paris, Hachette supérieur, 2005 aux pp.50 et s. [Bakouche].

7. Jacqueline Pousson-Petit, "Conclusion" dans Jacqueline Pousson-Petit, dir., L'identité de la personne humaine, étude de droit français et de droit comparé, Bruxelles, Bruylant, 2002, 979 [Pousson-Petit, "Conclusion"].

8. Supra note 6 à la p.51.

9. $\quad$ Voir sur ce point supra note 5. 


\section{A - L'identité civile saisie par la religion}

L'identité civile possède une fonction informative. Chaque élément qui compose l'état des personnes contribue à préciser le statut civil du sujet de droit, statut individuel et familial. Ces éléments sont caractérisés par leur indisponibilité et en principe soustraits à l'emprise de la volonté individuelle afin justement d'en assurer la stabilité dans un but de police. Cependant, une telle rigidité connaît des atténuations avec le développement de nouvelles fonctions de l'état civil que l'on peut qualifier de psychologiques et que le droit, fait nouveau, appréhende ${ }^{10}$.

Le prénom participe à la fonction sociale d'identification, mais de façon auxiliaire, complémentaire du rôle joué par le nom $^{11}$. En dehors du droit, le prénom a une valeur symbolique. Sa fonction juridique n'est que secondaire : l'importance (sociologique et psychologique) dont se charge le prénom montre qu'en réalité sa fonction principale est extrajuridique. Il est ce support "personnalissime" de la personnalité comme le qualifie le doyen Cornu. Le choix initial du prénom par les parents peut dans certains cas traduire un attachement à une foi et, dans cette perspective, révéler une part d'identité personnelle. Il se peut également qu'à un certain moment de sa vie, l'individu éprouve la nécessité d'une mise en correspondance de sa foi avec son prénom, celui-ci contredisant la religion réellement professée ou gênant son exercice. Cette mise en harmonie fonde alors une demande en changement de prénom à laquelle les tribunaux accèdent fréquemment ${ }^{12}$. Identité civile et identité religieuse se rejoignent alors dès lors que l'individu peut exciper d'un intérêt légitime à ce changement. Le système juridique permet cette

10. Daniel Gutmann, "Identité civile et identité familiale» Petites Affiches, Paris, 28 avril 1999, n. 84 à la p.37 [Gutmann, "Identité»]; Daniel Gutmann, Le sentiment d'identité, étude de droit des personnes et de la famille, coll. Bibliothèque de droit privé, Paris, L.G.D.J., t. 327, 2000 [Gutmann, "Sentiment»].

11. Gérard Cornu, Droit civil, Introduction, coll. Domat de droit privé, Montchrestien, 1995 à la p. 234 [Cornu, "Droit civil»].

12. Vincente Fortier, Justice, religions et croyances, coll. CNRS droit, Paris, 2000 aux pp. 98 et s. [Fortier, "Justice»]. 
(2008) 38 R.D.U.S.

Les incertitudes juridiques

de

l'identité religieuse

appropriation par le sujet de son identité religieuse, même s’il s'agit là d'une appropriation contrôlée.

Comme le remarque Daniel Gutmann, notre idéologie juridique veut que l'identité soit civile avant d'être familiale :

Traditionnellement, écrit cet auteur, la fonction première de l'état civil est d'identifier l'individu et de lui donner un moyen de preuve qu'il est bien celui qu'il prétend être. L'identité est alors la condition de l'intégration dans la cité. Pourtant, il est tout aussi vrai que l'identité civile désigne également une identité familiale. ${ }^{13}$

Par le jeu du mécanisme informatif (le nom qui révèle en principe une filiation, mais également le statut matrimonial), l'identité civile est le reflet de l'identité familiale et rattache l'individu à une généalogie. L'identité familiale résulte non seulement de la filiation, mais embrassant la situation matrimoniale et parentale, reflète tous les événements qui marquent la vie. L'identité familiale est très souvent investie par la religion. Le mariage, par exemple, comporte pour bon nombre de couples une dimension spirituelle certaine. Bien que le droit français confère une exclusivité au mariage civil et dénie toute autonomie au mariage religieux, il n'en demeure pas moins que le droit n'ignore pas totalement la religion : les solutions apportées par les juges au problème du Guett ${ }^{14}$, la considération certes bien timide des convictions religieuses dans le démariage ${ }^{15}$, l'existence même de la séparation de corps, si longtemps appelée divorce des catholiques, dans la mesure où elle ne porte pas atteinte au dogme de l'indissolubilité du mariage, bien que peu utilisée (5000 cas par an), mais maintenue par la loi du 26 mai 2004 portant réforme du divorce, témoignent de la prise en compte par le droit de l'identité religieuse dans le statut matrimonial. Toutefois, le droit est là aussi pour poser les limites à l'expression des

13. Gutmann, "Identité", supra note 10.

14. Vincente Fortier, "Le juge gardien du pluralisme confessionnel», RRJ, 2006-3 aux pp.1163 et s. [Fortier, «juge»].

15. Ibid. à la p. 1165. 
convictions religieuses lorsque celles-ci heurtent nos valeurs fondamentales. Deux exemples illustrent notre propos.

Le premier concerne la lutte contre les mariages forcés, l'un des buts que s'est assignée la loi du 4 avril $2006^{16}$ renforçant la prévention et la répression des violences au sein du couple ou commises contre les mineurs ${ }^{17}$. Certaines jeunes filles ou des jeunes hommes, français d'origine étrangère ou étrangers vivants en France sont mariés de force à des personnes qu'ils ne connaissaient pas ou à des membres de leur famille élargie à l'occasion de vacances dans le pays d'où leurs parents ont émigré18. Cette pratique concerne les familles originaires de Turquie, d'Asie, du Sénégal et aussi du Maghreb. Si la pratique ancestrale du mariage arrangé par les familles n'est pas critiquable en tant que telle, en revanche lorsque l'enfant n'est pas libre de consentir à cette union, il y a atteinte à un droit de l'homme fondamental, celui d'aimer et de se marier librement. Le nombre, en France, de victimes de tels mariages non consentis est difficile à estimer, car les jeunes gens hésitent à désobéir à leur famille en refusant l'union, en prenant la fuite ou en dénonçant l'union forcée à la justice. Certaines associations avancent le chiffre de 70000 mineures et majeures potentiellement concernées ${ }^{19}$. Même si ce chiffre est contesté, l'augmentation tendancielle du nombre de mariages forcés est une

16. Loi $n^{\circ}$ 2006-399 du 4 avril 2006, J.O., 5 avril 2006, 5097.

17. On notera que l'Assemblée parlementaire du Conseil de l'Europe a recommandé aux États membres de mettre sur pied une politique de lutte contre les mariages forcés et les mariages d'enfants : Conseil de l'Europe, Rec. 1723 [2005], 5 octobre 2005, Droit de la Famille, novembre 2005, $\mathrm{n}^{\circ} 11$, alerte 92.

18. Patrick Bloche et Valérie Pecresse, "L'enfant d'abord", Rapport nํㅜ 2832 de la Mission d'information sur la famille et les droits des enfants, 25 janvier 2006.

19. Virginie Larribau-Terneyre, "La pratique des mariages forcés révélée par la jurisprudence», commentaire sous Cour d'appel de Colmar, 28 avril 2005, Droit de la famille, janvier 2006, $\mathrm{n}^{\circ} 1$, comm. 1 [Larribau-Terneyre, "mariages"]; Virginie Larribau-Terneyre, "La loi contre la tradition : la lutte contre les mariages forcés par le relèvement de l'âge légal du mariage des femmes", Droit de la famille, mai 2005, $\mathrm{n}^{\circ} 5$, repère 5 [Larribau-Terneyre, «loi»]. 
réalité et doit être replacée dans le contexte de la progression des mariages mixtes célébrés à l'étranger qui sont de l'ordre de 45000 par an. S'inscrivant dans le cadre du plan global de lutte contre les violences faites aux femmes lancé par le gouvernement le 24 novembre 2004, la loi du 4 avril 2006 dans son volet civil est le reflet d'un ordre public matrimonial de protection renforcé 20 . D'une part, la loi modifie l'article 144 du Code civil pour unifier à dix-huit ans l'âge requis pour se marier. Outre de promouvoir le principe d'égalité ou encore de s'adapter aux réalités sociales en tenant compte davantage de l'âge de la maturité que de l'âge de la puberté, l'augmentation de l'âge pour les filles a pour but de renforcer la liberté du mariage en protégeant la liberté du consentement pour lutter contre la pratique des mariages forcés. En relevant l'âge du mariage des femmes à l'âge de la majorité, la loi entend donner aux filles davantage de moyens pour résister à la pression parentale et refuser l'union que la famille veut leur imposer ${ }^{21}$. Cependant, cette disposition ne protège que les jeunes filles âgées de 15 à 18 ans, les plus vulnérables certes, mais pas les seules à être concernées par le problème des mariages forcés. La Commission nationale consultative des droits de l'homme observe que des jeunes femmes mineures ou majeures, étrangères ou binationales peuvent se voir imposer des mariages contractés à l'étranger ou en France selon des règles coutumières qui permettent de sceller une union sans mariage civil, celui-ci étant célébré plusieurs années après. En outre, remarque-t-elle, le respect des prescriptions relatives à l'âge du mariage suppose l'existence de registres d'état civil tenus rigoureusement ce qui n'est pas le cas dans tous les pays. C'est pourquoi la loi du 4 avril 2006 renforce l'ordre public matrimonial en étendant le rôle du ministère public. Désormais, celui-ci peut surseoir à la célébration

20. Marc Azavant, "Regard civiliste sur la loi du 4 avril 2006 renforçant la prévention et la répression des violences au sein du couple», Droit de la famille, octobre 2006, $\mathrm{n}^{\circ} 10$, étude 40. À titre de comparaison, Christophe Le Berre, "Le cas des femmes qui entendent se soustraire à des mariages imposés", Droit administratif, juin 2005, n 6, comm. 89 (Commission de recours des réfugiés, 24 septembre 2004, ${ }^{\circ} 444000$, Mlle N.).

21. En 2002, 469 personnes de moins de 18 ans se sont mariées soit 460 jeunes filles dont deux de moins de 15 ans et neuf garçons de 17 ans. 
du mariage en cas de vice du consentement 22 , il peut également intenter une action en nullité relative pour vice du consentement ${ }^{23}$. La sanction de la violation de l'ordre public est encore renforcée par la prise en compte de la crainte révérencielle comme cause de nullité du mariage ${ }^{24}$. La jurisprudence admettait déjà que la contrainte familiale puisse constituer un vice de violence. Cependant, le contentieux est peu fourni puisque l'on dénombre, sur 15 ans, seulement 12 décisions, dont une seule de cassation concernant les mariages forcés alors que 78 sont relatives à des mariages de complaisance. Les cours n'ont prononcé que 6 annulations de mariage pour violence ${ }^{25}$. On peut bien évidemment douter de l'efficacité du nouveau texte. Il n'en demeure pas moins que "chaque interdit posé constitue une balise ou un kern, instrument d'une pédagogie de la citoyenneté» 26 . Même si certaines dispositions semblent n'avoir qu'une portée symbolique, elles améliorent tout de même la lisibilité des valeurs protégées dans notre société à l'égard des populations étrangères et jouent un rôle préventif et éducatif non négligeable ${ }^{27}$.

22. Art. 175-2 C.civ. Cette sanction a été jugée nécessaire car, en cas de mariage forcé, la contrainte persiste après la célébration du mariage, ôtant à l'époux dont le consentement a été vicié toute possibilité d'action (Anne-Marie Leroyer, "Loi $\mathrm{n}^{\circ}$ 2006-399 du 4 avril 2006 renforçant la prévention et la répression des violences au sein du couple ou commises contre les mineurs, Regard civiliste sur la loi relative aux violences au sein du couple», RTD Civ. 2006 à la p. 402).

23. Art. 180 C.civ.

24. En effet, l'article 180 dispose désormais que "l'exercice d'une contrainte sur les époux ou l'un d'eux, y compris la crainte révérencielle envers un ascendant, constitue un cas de nullité du mariage».

25. Larribau-Terneyre, "Mariages", supra note 19.

26. Christine Lazerges, "À propos des fonctions du nouveau Code pénal français", Archives de politique criminelle, $\mathrm{n}^{\circ} 17$ à la p.7.

27. On ajoutera que la loi du 26 novembre 2003 relative à la maîtrise de l'immigration, au séjour des étrangers en France et à la nationalité comporte des dispositions qui peuvent être utilisées dans le cas des mariages forcés. C'est le cas notamment de l'audition des futurs époux désormais obligatoire lorsqu'il existe un doute sur la réalité de leur consentement. Une circulaire du garde des Sceaux de mai 2005 dresse une liste indicative des indices faisant suspecter l'absence de liberté matrimoniale par exemple : l'un des conjoints est vulnérable; ou lors du dépôt du dossier ou de la cérémonie l'un des futurs époux est dans un 
Le second exemple illustrant les limites juridiques à l'irruption de la religion dans la vie familiale concerne la répudiation de droit islamique comme moyen juridique de rupture du mariage, et qui nous entraîne sur les terres du droit international privé28. La répudiation se caractérise par le pouvoir discrétionnaire reconnu à l'homme dans la dissolution du mariage et, en conséquence, par la situation très fragile dans laquelle se trouve la femme, non seulement du point de vue pécuniaire, mais également quant à la pérennité de son union conjugale. Comme le souligne Jean-Pierre Marguénaud,

on aura beau faire et beau dire: même enrobée des garanties procédurales les plus raffinées, même accompagnée des compensations financières les plus mirobolantes, la répudiation, dans la mesure où elle conduit à la dissolution du mariage par la volonté unilatérale du seul mari, reste l'institution discriminatoire par excellence ${ }^{29}$.

La question des répudiations a fait l'objet depuis vingt-cinq ans des plus vives discussions et suscité nombre de solutions jurisprudentielles 30 .

Pendant un temps, la jurisprudence de la Cour de cassation sembla se fixer sur des bases solides, celles des droits

état d'hébétude ou présente des traces récentes de coups, ou encore l'un des époux a fait des déclarations même rétractées sur des pressions subies du fait de tiers, de l'autre conjoint, de ses parents ou de ses proches.

28. Voir Marie-Claire Foblets, "Le statut personnel musulman devant les tribunaux en Europe. Une reconnaissance conditionnelle» dans PoussonPetit, "Conclusion", supra note 7.

29. Jean-Pierre Marguénaud, "Quand la Cour de cassation française n'hésite plus à s'ériger en championne de la protection des droits de la femme : la question de la répudiation", RTD Civ. 2004 à la p. 367 [Marguénaud].

30. Hugues Fulchiron, "Requiem pour la répudiation musulmane?», JCP 2004, éd G, n 36, II 10128; Stéphane David, note sous cass. 17 février 2004, Droit de la famille, $\mathrm{n}^{\circ}$ 298; Fanny Vasseur-Lambry, note sous Cour d'appel de Douai, 8 juin 2000, Petites Affiches, 9 octobre 2001, n 201 à la p. 16. 
fondamentaux: la répudiation est une institution inégalitaire puisque réservée au seul mari, elle heurte donc le principe d'égalité des époux affirmé par la $\mathrm{CEDH}^{31}$. Intrinsèquement contraire à l'ordre public français en matière internationale qui compte le principe d'égalité au titre de ses exigences, la répudiation ne peut être prononcée par le juge français; bien plus, une répudiation prononcée à l'étranger ne peut recevoir quelque effet que ce soit en France.

Cependant, en 1999 puis en 2001, la Cour de cassation renonça à raisonner en termes d'égalité entre époux. Prenant acte de ce que la répudiation est une institution fondamentale du droit familial musulman, la Cour de cassation ne prohiba que les répudiations abusives ${ }^{32}$. Ces solutions de compromis n'emportèrent pas la faveur de la doctrine et certaines cours d'appel résistèrent à ces solutions.

Le point apparemment final à ces hésitations jurisprudentielles en matière de répudiation a été marqué par les cinq arrêts rendus le 17 février 2004 par la Cour de cassation et qui par leur nombre, la fermeté de leur motivation et leur publicité relèvent incontestablement des arrêts de principe ${ }^{33}$. Ces arrêts portent condamnations des répudiations musulmanes et sont un véritable "tir de barrage" contre la répudiation. La condamnation est générale : elle vise tout autant la répudiation en la forme privée traditionnelle que les hypothèses de répudiations judiciarisées contemporaines. Elle s'applique dès lors qu'il y a répudiation

31. À l'article $5 \mathrm{du}$ protocole additionnel $\mathrm{n}^{\circ} 7 \mathrm{du} 22$ novembre 1984 à la convention EDH.

32. Celles qui n'étaient prononcées que pour échapper à la menace d'un divorce en France, celles qui bafouaient les droits de la défense ou encore celles qui n'assuraient pas à l'épouse des garanties financières minimales.

33. Cass. 1re civ., 17 fév. 2004, D. 2004, 815, chron., Patrick Courbe, et 824, concl. Francis Cavarroc; Gaz. Pal., 25-26 février 2004, 29, note MarieLaure Niboyet; RJ personnes et famille, mai 2004, 6, comm. Marie-Claude Meyzeaud-Garaud; dans le même sens, Cass. 1re civ., 20 septembre 2006, AJ Fam. 2007, 139; Cour d'appel de Paris, 12 octobre 2006, AJ Fam. 2007, 139 (note Alexandre Boiché). 
(2008) 38 R.D.U.S.

Les incertitudes juridiques

de

l'identité religieuse

stricto sensu, c'est-à-dire exercice d'un droit reconnu au mari de mettre fin au mariage par décision unilatérale, sans contrôle judiciaire réel sur les motifs ou sur l'opportunité de la rupture. La solution se fonde sur la contrariété intrinsèque de cette institution au principe d'égalité entre homme et femme ${ }^{34}$, dès lors que la femme, sinon les deux époux, sont domiciliés sur le territoire français.

La solution retenue par la Cour de cassation $^{35}$ peut contribuer à la politique d'intégration des étrangers, elle peut également renforcer la position de tous ceux qui, dans les pays musulmans, se sont engagés dans des mouvements progressistes et luttent contre des institutions archaïques, pour la modernisation de ces sociétés ${ }^{36}$. Ainsi, en est-il du Maroc qui, dans le Code de la famille, a instauré le principe d'égalité entre les époux le 23 janvier 2004.

\section{B - L'identité corporelle saisie par la religion}

"Le corps est le temple de l'Esprit-Saint", nous dit saint Paul dans l'Épître aux Corinthiens ${ }^{37}$. Le corps humain n'est pas une chose : il est la personne même ${ }^{38}$. Considéré par les anthropologues comme la souche identitaire de l'homme, le corps humain est tout à la fois un moyen de s'identifier (en tant que support matériel de la personnalité d'un individu) et d'être identifié comme croyant, le corps étant apparence, il véhicule des

34. Ce principe est garanti par l'article $5 \mathrm{du}$ protocole $\mathrm{n}^{\circ} 7$ de la convention EDH et partie intégrante de l'ordre public français en matière internationale,

35. Voir cependant les réserves émises par Marguénaud, supra note 29, qui considère qu'il faut se garder d'une approche trop dogmatique de la question et qu'il faut "éviter que le dynamisme propre aux droits fondamentaux (leur caractère hégémonique diraient certains) ne détruise les équilibres subtils élaborés par la doctrine et la jurisprudence pour concilier les différents impératifs du droit international privé».

36. Patrick Courbe, supra note 33.

37. Saint Paul, Épître aux Corinthiens, chapitre VI, verset 19.

38. Cornu, "Droit civil", supra note $11, n^{\circ} 479$. 
informations personnelles sur un individu et se fait aux yeux d'autrui le révélateur d'une croyance religieuse ${ }^{39}$.

Le corps est conducteur de symboles. Depuis que la loi mosaïque a institué la circoncision comme signe de l'Alliance entre l'homme juif et son Dieu, depuis que le fait d'être circoncis a pu devenir pendant le génocide de la Seconde Guerre mondiale, signe de mort, on sait comment une marque sur le corps peut inscrire le symbolique dans la chair vive ${ }^{40}$.

Lorsqu'elle s'exprime par le corps, la relation au religieux comporte deux dimensions : l'une éclatante, lorsque privilégiant l'apparence du corps, la religion se donne à voir. Et nous pensons ici bien évidemment au port d'un signe religieux visible, que celuici s'analyse comme une obligation intrinsèque à la religion professée ou comme une manifestation d'appartenance. L'autre dimension est intime, liée à des situations personnelles qui touchent à la vie privée. Face aux marqueurs religieux supportés par le corps ou inscrits dans le corps, quelle est l'attitude du droit?

Entre tolérance, exclusion et protection, les solutions de droit oscillent.

La tolérance s'exerce à l'égard de la circoncision rituelle, pourtant véritable exception au principe d'intangibilité du corps humain puisque la pratique consomme une atteinte irréversible de celui-ci. Elle constitue incontestablement une atteinte à l'intégrité $\mathrm{du}$ corps humain, en totale contradiction avec l'article 16-3 du Code civil qui dans sa rédaction issue de la loi du 6 août 2004 dispose :

Il ne peut être porté atteinte à l'intégrité du corps qu'en cas de nécessité médicale pour la personne ou à titre exceptionnel dans l'intérêt thérapeutique d'autrui.

39. Voir également supra note 5 aux pp. 75 et s.; Philippe Dubois, Le physique de la personne, Paris, Economica, 1986, 354.

40. Catherine Clément, "Circoncision et excision", Encyclopaedia Universalis. 
La circoncision est une mutilation sexuelle et son fondement religieux n'y change rien. À ce titre, elle devrait encourir les foudres du droit pénal. Or elle n'est jamais poursuivie. Plus encore, aucun obstacle ne s'est jamais élevé contre la circoncision rituelle. On avancera le fait que la circoncision des juifs s'est pratiquée depuis toujours en France, que celle des musulmans en Afrique du Nord n'a pas ému l'administration coloniale et qu'aujourd'hui, le rite est perpétué dans notre pays sans clandestinité ${ }^{11}$. Le corps médical ne s'y oppose pas et la pratique est pour les musulmans effectuée à l'hôpital, semble-t-il remboursée par la sécurité sociale codifiée sous le sigle correspondant à l'opération du phimosis.

Y aurait-il alors une permission coutumière à l'égard de la circoncision? C'est discutable ${ }^{42}$. Quoi qu'il en soit, on peut d'un point de vue fondamental se demander si une coutume (si coutume il y a) pourrait couvrir une infraction aussi grave que les mutilations du corps.

Ordinairement couverte par le secret des familles, la circoncision se retrouve sur la scène judiciaire en deux occasions : d'une part, lorsque le père fait circoncire son ou ses fils sans avoir requis au préalable le consentement de la mère dont il est séparé (cela intéresse surtout les couples mixtes); d'autre part, lorsque les suites de la circoncision connaissent des complications qui peuvent être particulièrement graves allant jusqu'au décès de l'enfant. Même si ces derniers cas sont rares, ils montrent toutefois que l'acte est loin d'être anodin.

L'examen de la jurisprudence la plus récente montre que cette tolérance manifestée à l'égard de la circoncision est sans doute un peu moins bienveillante qu'auparavant. Qualifiée "d'acte grave ou encore d'acte important et unique dans la vie de l'enfant, qui n'est pas courant en France, d'acte non usuel de l'autorité

\footnotetext{
41. Christine Choain, note sous Cass. 1re civ., 26 janvier 1994, D. 1995. jurispr., 226.

42. Ibid.
} 
parentale" par la jurisprudence ${ }^{43}$, la circoncision impose au père de requérir le consentement de la mère ${ }^{44}$. Sa carence en ce domaine est un motif grave qui peut entraîner la suppression de son droit d'hébergement voire de son droit de visite ${ }^{45}$. Par ailleurs, cette qualification de l'acte oblige le médecin à s'assurer du consentement des deux parents. Si tel n'est pas le cas, il fera preuve d'une légèreté blâmable et donc sa responsabilité pourra être engagée ${ }^{46}$. En fait, ce sont davantage les conditions de sa réalisation que la circoncision elle-même que le droit sanctionne. Et pourtant, la circoncision rituelle, geste irréversible et discriminant, ne fait pas que guider l'enfant dans le choix d'une religion, prérogative parentale, mais plutôt lui impose pour la vie entière une inscription religieuse dans son corps.

L'exclusion, à l'inverse, frappe l'excision, ce rite initiatique qui marque un changement de la personne, mais mutilation barbare qu'aucune croyance, fût-elle religieuse ne saurait justifier ${ }^{47}$. Pour ne nous en tenir qu'à la France, on estime qu'au moins 30000 femmes et fillettes excisées vivent actuellement sur notre territoire. Différentes organisations avancent le chiffre de 10 000 à 20000 petites filles originaires d'Afrique qui seraient exposées au risque d'excision ${ }^{48}$. La France est le seul pays d'Europe où l'excision a déjà donné lieu à plus de vingt procès. L'excision tombe sous le coup de la loi pénale : l'article 222-9 du

43. Cour d'appel de Rennes, 4 avril 2005, jurisdata ${ }^{\circ}$ 2005-292420; Cour d'appel de Paris, 29 septembre 2000, D. 2001, p. 1585, note Cyrille Duvert.

44. Cour d'appel de Paris, 13 septembre 2000, jurisdata $\mathrm{n}^{\circ}$ 2000-125029; Cour d'appel de Rennes 4 avril 2005, précit.; Cour d'appel d'Orléans, 14 mars 2006, jurisdata $\mathrm{n}^{\circ}$ 2006-310764. Contra : Cour d'appel d'Aix-enProvence, 4 mars 2004 (concernant deux époux de confession musulmane), jurisdata $\mathrm{n}^{\circ}$ 2004-238032.

45. Par exemple, Cour d'appel de Poitiers, 21 novembre 2000, jurisdata n² 2000-146397.

46. Cour d'appel de Paris, 29 septembre 2000, précit.; Thierry-Xavier Girardot, "Régime de la responsabilité sans faute des établissements publics d'hospitalisation", AJDA 1997 à la p. 959.

47. Supra note 12 aux pp. 39 et s.; supra note 5 aux pp. 78 et s.

48. Jean-Charles Champagnat, "Les droits de l'enfant" (1999-2008), en ligne : Les droits de l'enfant <www.droitsenfant.com>. 
(2008) 38 R.D.U.S.

Les incertitudes juridiques

de

l'identité religieuse

Code pénal punit les violences ayant entraîné une mutilation de dix ans d'emprisonnement et de $150000 €$ d'amende. L'infraction est criminalisée dès lors que l'excision est pratiquée sur un mineure de quinze ans: elle est passible de quinze ans de réclusion criminelle et, si elle a été commise par les parents, la peine est portée à vingt ans de réclusion. Lorsque ces violences entraînent la mort de l'enfant (ce qui a déjà été le cas en France), l'article 222-8 prévoit une peine d'une durée de trente ans de réclusion criminelle. Cependant, et malgré la criminalisation de l'infraction depuis 1984, les sanctions apparaissent seules impuissantes à éradiquer une pratique identitaire au fondement religieux très contestable. C'est que la loi du silence règne autour de l'excision vécue par ceux qui la pratiquent comme une coutume ancestrale et donc contraignante. Effet pervers de la répression : les parents font pratiquer l'excision sur leurs fillettes dans leur pays d'origine durant les vacances. Mais la loi pénale s'applique à eux de la même manière. On notera également qu'en juin 2004, l'Académie de médecine a rappelé aux médecins qu'ils devaient signaler tout cas d'excision constaté. Certains avancent que la condamnation de l'excision en France serait la manifestation d'un néocolonialisme culturel. L'argument n'est pas recevable, l'acte emportant pour la fillette excisée de telles conséquences qu'il n'est pas admissible.

Exclusion encore, sur d'autres fondements, du port d'un signe religieux manifestant ostensiblement une appartenance religieuse par les élèves dans les écoles de la République ${ }^{49}$ ou par les agents de l'État dans l'exercice de leurs fonctions ${ }^{50}$.

Mais protection vigoureuse du croyant, victime d'une discrimination en raison de sa religion. Tel est le cas par exemple

49. Loi $n^{\circ}$ 2004-228 du 15 mars 2004 encadrant, en application du principe de laïcité, le port de signes ou de tenues manifestant une appartenance religieuse dans les écoles, collèges et lycées publics, J.O., 17 mars 2004, 5190.

50. Voir sur ce point Joël Berthoud, "La neutralité religieuse du fonctionnaire", Semaine juridique Administrations et collectivités territoriales, 21 mars 2005, n 12 à la p. 1142 . 
en droit social avec l'article L.1132-1 qui interdit toute mesure à l'encontre du salarié prise en considération de ses convictions religieuses ${ }^{51}$.

\section{II - Revendiquer son identité religieuse pour afficher sa foi}

L'identité comporte deux signifiés: celui qui recouvre l'identité individuelle, son versant individualiste et que nous venons d'évoquer et celui qui permet l'identification à la communauté d'appartenance, le versant communautaire de l'identité52. C'est à celui-ci que nous allons désormais nous attacher. Comme le souligne le sociologue Gérard Neyrand,

l'accroissement de la réflexivité individuelle dans les sociétés occidentales a contribué à l'émergence de revendications spécifiantes concernant certaines caractéristiques individuelles qui représentent pour le sujet un enjeu identitaire fort. La cristallisation de ces revendications en mouvements sociaux (...) a symbolisé le passage à un nouveau régime identitaire, celui de l'affirmation volontaire de noyaux organisateurs choisis dans la diversité des références constitutives de l'identité personnelle, qui jouent le rôle de principes structurants d'une identité vécue comme trop éclatée. ${ }^{53}$

Évoquer le versant communautaire de l'identité, c'est renvoyer à la notion de communautarisme et, par voie de conséquence, à la mise en avant de l'appartenance à une communauté laquelle revendique d'être reconnue, entendue en tant que telle.

51. Pour de plus amples développements, nous renvoyons à notre article, Fortier, "Juge", supra note 14 aux pp. 1167 et s.

52. "Toutes les revendications identitaires sont d'ailleurs intrinsèquement paradoxales, elles possèdent un versant individualiste déjà équivoque mais aussi un versant communautaire. Ces deux logiques peuvent entrer en conflit": Pousson-Petit, "Conclusion", supra note 7 à la p. 984 .

53. Gérard Neyrand, "Identification sociale, personnalisation et processus identitaires", dans Jacqueline Pousson-Petit, L'identité de la personne humaine, étude de droit français et de droit comparé, supra note 7 aux pp. 93 et $\mathrm{s}$. 
(2008) 38 R.D.U.S.

Les incertitudes juridiques

de

403

l'identité religieuse

En France, le communautarisme est perçu négativement : la reconnaissance de l'appartenance favoriserait un système de personnalité des lois, c'est-à-dire de pluralisme juridique extrême laissant craindre une "tribalisation" de la société, une remise en cause du lien social, à tout le moins sa fragilisation ${ }^{54}$. On peut retenir comme définition du communautarisme, celle qui y voit un affaiblissement de la prise en compte abstraite de l'individu au profit de la surévaluation de certains de ses traits physiques, ethniques, sexuels, religieux, l'essentiel étant qu'ils soient considérés comme débouchant sur une vision du monde particulière commune à ceux qui les ont en partage ${ }^{55}$. L'individu est alors réduit à telle particularité et demande une réorganisation de son statut en fonction de la culture ainsi induite. Le communautarisme exalte l'individu, mais après l'avoir fondu dans le groupe ${ }^{56}$. Mais on peut penser le communautarisme, c'est-à-dire la reconnaissance des appartenances "non point comme une invitation au repli des communautés sur elles-mêmes, ni comme une apologie de la différence pour la différence», mais comme la possibilité de "concevoir un droit commun qui, par exemple au moyen de la dialectique du principe et de l'exception, soit apte à accueillir une diversité reconnue comme légitime par le corps politique»57.

Les tribunaux n'échappent pas aux manifestations de ce communautarisme, et les revendications fondées sur une reconnaissance de la singularité d'un groupe nourrissent une part $\mathrm{du}$ contentieux. Ces revendications lorsqu'elles sont de nature religieuse, illustrent un retour ou une poussée vers l'orthodoxie qui ne va pas sans heurter le droit. Dans un tel cas de figure, la règle religieuse est en quelque sorte surexposée et, dans sa prétention normative, vient concurrencer, jusqu'à chercher à la supplanter, la loi commune. "Le communautarisme se traduit par

\footnotetext{
54. Sur cette question, nous renvoyons à l'analyse pénétrante de Daniel Gutmann, "sentiment", supra note 10 aux pp. 434 et s.

55. Rémy Libchaber, "Aspects du communautarisme : fait et droit religieux au regard du droit», RTD Civ. 2003 à la p. 575 [Libchaber].

56. Ibid.

57. Gutmann, "Sentiment", supra note 10 à la p. 4.
} 
une projection des singularités de la personne sur son environnement juridique et par une soumission des droits qui doivent lui être octroyés à ses besoins identitaires" ${ }^{58}$.

Quelques affaires récentes en droit des contrats fournissent un exemple particulièrement topique de la situation. Elles invitent à s'interroger sur la légitimité d'une demande tendant à la prise en compte de l'appartenance religieuse pour déroger à la règle commune.

La première affaire $^{59}$ concerne un groupe de vingt-cinq locataires de confession juive habitant un ensemble immobilier de mille personnes. Pour améliorer la sécurité de la résidence à la demande d'une association de locataires, la société propriétaire de l'ensemble a fait installer autour de la résidence des grilles et quelques portes s'ouvrant grâce à un clavier électronique (digicode) qui enclenche un dispositif électronique. Or, les vingtcinq locataires observateurs scrupuleux des prescriptions de leur religion leur interdisant l'utilisation de toute forme d'énergie et notamment, l'électricité, assimilée au feu, pendant le shabbat (donc du vendredi soir au samedi soir) et les fêtes religieuses se sont insurgés contre la mise en place d'un dispositif qui, certains jours, les obligeait à choisir entre une violation de l'interdit électrique et une absence aux offices religieux.

Les locataires assignèrent alors la société en vue d'obtenir l'installation d'une serrure mécanique et la remise des clefs permettant ainsi d'éviter l'utilisation du digicode litigieux. La Cour de Paris, par son arrêt du 27 octobre 200060, accueillit favorablement la demande en se fondant sur la liberté de culte garantie par la Constitution française et sur les textes internationaux sans précision, mais parmi lesquels on peut

58. Ibid.

59. Cass. 3e civ., 18 décembre 2002, RJPF, avr. 2003 à la p. 9 (note Éric Garaud), RTD civ. 2003 à la p. 90, obs. Jacques Mestre et Bertrand Fages à la p. 383, obs. Jean-Pierre Marguénaud à la p. 575, obs. Rémy Libchaber, AJDI 2003 à la p. 182, concl. Olivier Guérin.

60. Cour d'appel de Paris, 27 octobre 2000, Jurisdata n 2000-128442. 
(2008) 38 R.D.U.S.

Les incertitudes juridiques

de

405

l'identité religieuse

supposer bien évidemment l'article 9 de la CEDH. Confirmant la décision des premiers juges, elle considère que le fait de refuser d'installer des serrures mécaniques au moins pour l'un des accès à la résidence cause un trouble manifestement objectif aux résidents qui en font, dit la Cour, la demande légitime. Sous le visa de l'article 9 de la CEDH et de l'article 1134 du Code civil, la Cour de cassation dans un attendu cinglant casse l'arrêt de la Cour de Paris :

Les pratiques dictées par les convictions religieuses des preneurs n'entrent pas, sauf convention expresse, dans le champ contractuel du bail et ne font naître à la charge du bailleur aucune obligation spécifique.

Cette décision peut être analysée à un triple point de vue juridique correspondant du reste au triple visa de l'arrêt de cassation : l'article 1134 du Code civil selon lequel les conventions doivent être exécutées de bonne foi, la CEDH dans ses articles 9-1 et $9-2^{61}$ qui protègent la liberté religieuse, la liberté de manifester sa religion et d'exercer son culte, et l'article 6 de la loi du 6 juillet 1989 concernant le bail d'habitation. Sans entrer dans la technique juridique, il apparaît toutefois que plusieurs enseignements doivent être tirés de la solution de la Cour.

À la question de savoir si au nom du respect des droits fondamentaux du contractant, il fallait considérer que la religion s'était intégrée dans la loi du contrat, la Cour régulatrice oppose un principe de laïcité contractuelle, affirmé avec force. En dehors d'une contractualisation expresse de l'élément religieux, les pratiques religieuses n'entrent pas dans le champ du contrat. En cela, la solution suit une jurisprudence bien établie, en matière de droit du travail: depuis 1998, au moins, la chambre sociale considère que les convictions religieuses du salarié n'entrent pas, sauf clause expresse, dans le cadre du contrat de travail 62 .

61. Voir sur ce point les observations de Marguénaud, supra note 29.

62. Cass. soc., 24 mars 1998, Bull. civ. V, n¹71, Dr. Soc. 1998, 614, obs. Savatier; Yves Chauvy, "Travail et religion sous le rapport tempéré de la 
Par ailleurs, "les pratiques dictées par les convictions religieuses" selon les termes de la Cour de cassation, ne peuvent faire irruption dans le contrat au moment de son exécution par le biais de l'exigence de bonne foi. Elles ne font naître, dit la Cour, aucune obligation spécifique. Cela signifie qu'une partie, même tenue d'exécuter loyalement le contrat, n'a pas le devoir d'en adapter le contenu aux préceptes et interdits religieux qu'observe son partenaire contractuel ${ }^{63}$.

Cette solution appelle, nous semble-t-il, quelques réserves : tout d'abord, et quelle que soit la décision des juges, la demande des locataires n'était-elle pas recevable dans son principe ${ }^{64}$ ? Ceuxci avaient pris à bail des appartements sans digicode; en l'installant, le bailleur modifiait quelque peu les conditions d'exécution du contrat. On peut bien sûr discuter sur les notions d'intérêt collectif et d'intérêt individuel; on peut regretter également qu'une médiation n'ait pas été mise en place qui aurait permis de trouver une solution conciliant les intérêts en présence. Sans doute aussi, la réception de cette sorte d'objection de conscience pourrait effectivement avoir des conséquences pratiques difficilement maîtrisables65. Cependant, ce qui est gênant dans l'attendu de la Cour de cassation, c'est que la revendication des locataires semble ne pas être recevable à raison même de son origine religieuse.

Cette affirmation catégorique d'un tel principe de laïcité contractuelle n'est pas forcément convaincante et il ressort que la Cour régulatrice fait peu de cas de la liberté de conscience. La même critique peut encore être formulée à l'encontre d'un arrêt rendu par la Cour de cassation le 8 juin 200666. Cet arrêt confirme que la Haute juridiction entend s'en tenir à l'application stricte des

\footnotetext{
neutralité (autour d'un interdit alimentaire dans l'islam d'outre-mer français)", Gaz. Pal., 18 novembre 1998, chron., 3.

63. Jacques Mestre, obs. supra note 59.

64. $\quad$ En ce sens voir Libchaber, supra note 59.

65. Voir les conclusions de Olivier Guérin, supra note 59.

66. Cass 3e civ, 8 juin 2006, Petites affiches, 5 juillet 2006, nº133, 9 (note Dominique Fenouillet).
} 
(2008) 38 R.D.U.S.

Les incertitudes juridiques

de

407

l'identité religieuse

règles, en l'espèce la force obligatoire des actes juridiques, peu important les impératifs de la conscience individuelle.

Il s'agissait ici d'un couple de copropriétaires, de confession juive qui avait édifié sur son balcon une construction en végétaux à l'occasion de la fête des cabanes. Prévue par la Torah, cette fête qui prescrit de construire des cabanes de feuillage et de bois et d'y habiter pendant sept jours, commémore les années d'errance du peuple juif dans le désert, et marque la confiance en Dieu et l'indifférence à l'aisance matérielle. Par une résolution votée en assemblée générale de copropriété, le syndic est mandaté pour agir en justice afin d'obtenir l'enlèvement de la cabane litigieuse. Les époux répliquèrent en assignant le syndicat des copropriétaires en annulation de la résolution. Ils estimaient que l'édification sur leur balcon pendant une semaine d'une cabane précaire et temporaire leur permettant de respecter les prescriptions de la religion juive, sans créer de nuisances ou de risques pour les autres copropriétaires, était conforme à la destination de l'immeuble. La résolution de l'assemblée générale devait dès lors être annulée comme restreignant leur droit d'exercice d'un culte sans être justifiée par la destination de l'immeuble. La Cour d'appel d'Aix-en-Provence ${ }^{67}$ rejeta la demande des époux : la cabane ne constituait pas un abri de jardin (permis par le règlement de copropriété), elle était constituée de parois de canisses (interdit par le même règlement) et elle portait atteinte à l'harmonie générale de l'immeuble, car visible de la rue. Enfin, "la liberté religieuse, pour fondamentale qu'elle soit, ne peut avoir pour effet de rendre licites les violations des dispositions d'un règlement de copropriété». Les époux formèrent un pourvoi en cassation. Ils reprochaient notamment à la Cour d'appel d'avoir violé l'article $9 \mathrm{du}$ Code civil et l'article 9 de la CEDH en permettant à un règlement de copropriété de priver un copropriétaire de sa liberté d'exercer son culte. La décision rendue par la Cour de cassation est intéressante en ce que, comme l'écrit Dominique Fenouillet, la liberté religieuse était soumise à

67. Cour d'appel d'Aix-en-Provence, 18 janvier 2005, jurisdata $\mathrm{n}^{\circ} 2005-$ 25078. 
l'épreuve du droit des biens, plus particulièrement aux dispositions d'un règlement de copropriété, cet acte juridique original de conciliation de l'individuel et du collectif. La Cour de cassation rejeta le pourvoi reprenant à son compte la formulation de l'attendu de la Cour d'Aix-en-Provence : la liberté religieuse, pour fondamentale qu'elle soit, dit la Cour de cassation, ne pouvait avoir pour effet de rendre licites les violations des dispositions d'un règlement de copropriété, la cabane faisant partie des ouvrages prohibés par le règlement et portant atteinte à l'harmonie générale de l'immeuble puisqu'elle était visible de la rue. Si la solution retenue en l'espèce n'était pas déraisonnable, en revanche elle traduit une certaine défiance à l'encontre de la liberté religieuse. Sans doute la solution se comprend-elle face à la progression des revendications identitaires de nature religieuse, du communautarisme qui peut affecter l'unité de la société. Le rappel des principes essentiels de notre droit est certes la mission de la Cour régulatrice. Mais la paix sociale ne s'est-elle pas construite sur un vaste compromis ${ }^{68}$ ? Une approche dogmatique de la laïcité est-elle la mieux à même de répondre aux revendications communautaires? Or, comme le remarque JeanMichel Belorgey,

on ne viendra à bout de ce que ces revendications identitaires ont de belliqueux et de menaçant pour la cohésion sociale, de l'ethnicisation redoutée, qu'en éliminant les comportements qui les engendrent; autrement dit en faisant une juste place à la différence, à

68. Michel Miaille, "À propos de citoyenneté et laïcité : débat sur l'universel", dans Véronique Champeil-Desplats et Nathalie Ferré, dir., Frontières $d u$ droit, critique des droits, Billets d'humeur en l'honneur de Danièle Lochak, coll. Droit et société, Paris, L.G.D.J., 2007 à la p. 83 : "force est de considérer aujourd'hui d'abord que les choses ont été plus complexes que cette histoire quelque peu enchantée d'une République radicale aux accents de l'abbé Grégoire rejetant les patois et les histoires locales et d'une séparation stricte en 1905 [...]. Après l'échec du projet Combes, la séparation version Briand est un compromis accentué par la pratique administrative marquée par l'œuvre du Conseil d'État. Les exceptions dans le droit - les statuts dérogatoires - comme dans les faits montrent que la France est loin du divorce célébré souvent de manière mythologique". 
(2008) 38 R.D.U.S.

Les incertitudes juridiques

de

409

l'identité religieuse

sa désignation, aux stratégies que son acceptation, et, dans une certaine mesure sa reconnaissance, devraient gouverner. 69

Cependant, le droit sait parfois faire une place au fait religieux en tant qu'expression d'un différentialisme porté par une communauté. Tel est le cas des entreprises de tendance. Encore que depuis $1991^{70}$, on note que la Cour de cassation a infléchi sa position à l'égard de ces entreprises, caractérisées par un projet qui promeut une doctrine, une idéologie, une morale particulière, dans un sens plus soucieux de la protection de la vie privée des salariés ${ }^{71}$. En effet, ces entreprises, selon l'arrêt Fischer de $1986^{72}$, étaient en droit d'exiger de leurs salariés "une communauté de pensée et de foi avec l'employeur", solution dont le fondement résultait de l'objet même de l'entreprise. L'évolution de la jurisprudence après cet arrêt est caractérisée par la recherche d'un équilibre entre les exigences propres à l'entreprise de tendance et la protection de la vie privée du salarié. En effet, si le comportement d'un salarié travaillant dans une entreprise de tendance peut fonder un licenciement, désormais c'est à la condition que, compte tenu de ses fonctions et de la finalité propre de l'entreprise, un tel comportement ait créé un trouble caractérisé au sein de l'entreprise. Telle est la solution adoptée depuis 1991 qui, marquant l'abandon de la jurisprudence Fischer, privilégie l'analyse objective des faits et l'application du principe de proportionnalité. Cependant, ce serait méconnaître la réalité de l'entreprise de tendance que de croire que le salarié n'est jamais tenu d'adhérer au projet religieux de l'entreprise pour laquelle il

69. Jean-Michel Belorgey, "Communautarisme ou universalisme: quel modèle?», dans Véronique Champeil-Desplats et Nathalie Ferré, dir., ibid. à la p. 27.

70. Cass. soc., 17 avril 1991, JCP 1991, éd. G, II, 21724, note Alain Sériaux.

71. Philippe Wacquet, "Loyauté du salarié dans les entreprises de tendance", Gaz. Pal. 1996, doctr. à la p. 1427.

72. Cass. soc., 20 novembre 1986, JCP 1987, éd. G, II 20798 (note Thierry Revet), et dont la solution est plus sévère que celle posée quelques années auparavant par l'Assemblée plénière dans l'affaire bien connue de la dame Roy et de l'association Sainte Marthe : 19 mai 1978, D. 1978, jurispr., 541 (concl. Robert Schmelck, note Philippe Ardant). 
travaille. Notamment pèse sur lui une obligation d'adopter une attitude qui ne contredise pas l'impératif religieux, ce qui parfois déborde le cadre de la vie professionnelle. Comme le soulignent certains auteurs, "dans l'entreprise de tendance, l'article L. 122-45 du Code du travail qui sanctionne les discriminations est en quelque sorte appliqué à rebours, à savoir que les choix de l'employeur (d'embaucher, d'affecter à un poste, de licencier) reposent sur l'idée de conformité religieuse ou de préférence, ce qui est illégal dans l'entreprise ordinaire»73. En revanche, il n'est pas possible d'admettre une surexposition de l'élément religieux dans l'entreprise de tendance qui conduirait à reconnaître implicitement la possibilité de supprimer ou de limiter les libertés du salarié à raison de l'objet même de l'entreprise.

À cette vigilance du droit à l'égard du fait communautaire religieux, plusieurs justifications peuvent être avancées qui plongent leurs racines dans notre histoire. On sait le long combat en France pour construire une sphère publique émancipée des appartenances privées et notamment religieuses. Et, du reste si l'identité civile peut être investie par la religion sans que le droit de manière générale n'y trouve pas grand-chose à redire, en revanche, dès lors que l'identité sociale est en cause, le droit affirme sa prééminence. Cette méfiance du droit s'explique également "par cette menace qui pèse toujours sur l'État d'être opposé à une organisation rivale» 74 .

Dans le fond, notre société est confrontée à un double changement de paradigme :

73. Henri Gleizes et Pierre Strasser, "Religion et travail" dans Francis Messner, Pierre-Henri Prélot et Jean-Marie Woehrling, dir., Traité de droit français des religions, Paris, Litec, 2003 à la p. 21.

74. Libchaber, supra note 55 ("Car les religions proposent du monde non seulement une vision structurée mais aussi et surtout un véritable ordre juridique. Dans ses relations avec les religions, l'État est contraint de raisonner de puissance à puissance. Cela explique qu'en France le traitement de la plupart des religions est non pas juridique mais politique. Cela conduit l'État à affirmer ses positions et l'incite à une hostilité de principe»). 
(2008) 38 R.D.U.S.

Les incertitudes juridiques

de

l'identité religieuse

- d'une part celui qui affecte les croyances plus intransigeantes vis-à-vis de l'extérieur sur le chapitre de la reconnaissance et qui, instrumentalisées, deviennent le vecteur de revendications communautaires.

- d'autre part, une diversité religieuse sans précédent qui s'est affirmée en un siècle.

L'enjeu du débat, nous semble-t-il, c'est la capacité de la République de composer avec le pluralisme religieux, pour assurer la paix sociale. 
